\title{
ARTHRITIS OF HIP COMPLICATING OSTEITIS PUBIS
}

\author{
H. B. Coates Milsom and B. S. Rose, Rotorua, New Zealand
}

From the Queen Elizabeth Hospital, Rotorua

A previously healthy man of seventy-four years, a retired farmer, was admitted to another hospital for prostatectomy in July 1954. At that time he had had symptoms of prostatic disease for over four years. A large adenomatous prostate was removed by Millin's retropubic method, and both vasa were tied. There had been a small quantity of residual urine before operation, but it was clear and was not cultured. Progress after the operation was satisfactory for the first few days, but he then suffered a secondary haemorrhage with high fever. The fever was accompanied by a persistent watery diarrhoea, which caused a marked deterioration in the patient's general condition.

One month after the operation he began to have pain in the pubic region, and radiographs showed osteitis pubis. As his general condition was improving he was then discharged after nine weeks in hospital.

After his discharge the pain eased in the pubic region, but spread laterally to the ischial tuberosities, where it became so severe that for several months he was unable to sit in one position for more than a few minutes. He could walk without discomfort until eight months after the operation, when his right hip joint became involved as the pain left the ischial tuberosities. The hip then rapidly became so severely affected that he was unable to walk, or even to move in bed without severe pain.

Nine months after the prostatectomy he was admitted to the Queen Elizabeth Hospital for Rheumatic Diseases at Rotorua with a diagnosis of arthritis of the right hip. At that time he was afebrile, but had to stay in bed for several weeks because of the painful hip. Review of the previous radiographs showed, in the first (undated) film, a normal appearance of the symphysis pubis and hip joint (Fig. 1); but in the second film taken eight months after the prostatectomy, the symphysis pubis appears fused, and there were signs of osteitis and periostitis of the ischio-pubic rami, with early spread to the right hip joint (Fig. 2).

Treatment and progress - The hip was rested at first with sustained traction, and later in a plaster hip spica. He remained comfortable in this for about three months, when he again developed a febrile illness with diarrhoea, and passage of mucopus from which none of the usual intestinal pathogens could be isolated. He failed to respond to streptomycin and aureomycin therapy, but the diarrhoea eventually subsided during treatment with succinyl sulphathiazole. Thereafter he suffered the further complication of left basal pneumonia with pleurisy and pericarditis and was in poor condition for several weeks. During this time the arthritis assumed secondary importance, and nothing further was done for it except to maintain fixation in the hip spica. His recovery was further delayed by an infected ulcer on the right foot, but after a total period of about six months' immobilisation the plaster spica was removed and he was encouraged to walk.

During the course of the illness the right hip joint had continued to show progressive destructive changes, with migration of the acetabulum upwards and outwards, and absorption of the femoral head (Figs. 3 to 5). The patient was finally left with a reasonable range of movement, like that after an excision arthroplasty.

\section{DISCUSSION}

Osteitis pubis is a self-limiting disease that seldom spreads beyond the pubic bones. On rare occasions it may, as in our patient, spread to involve the hip joint. Such a case was described by Friedenberg (1950), who was able to find records of two previous cases reported 


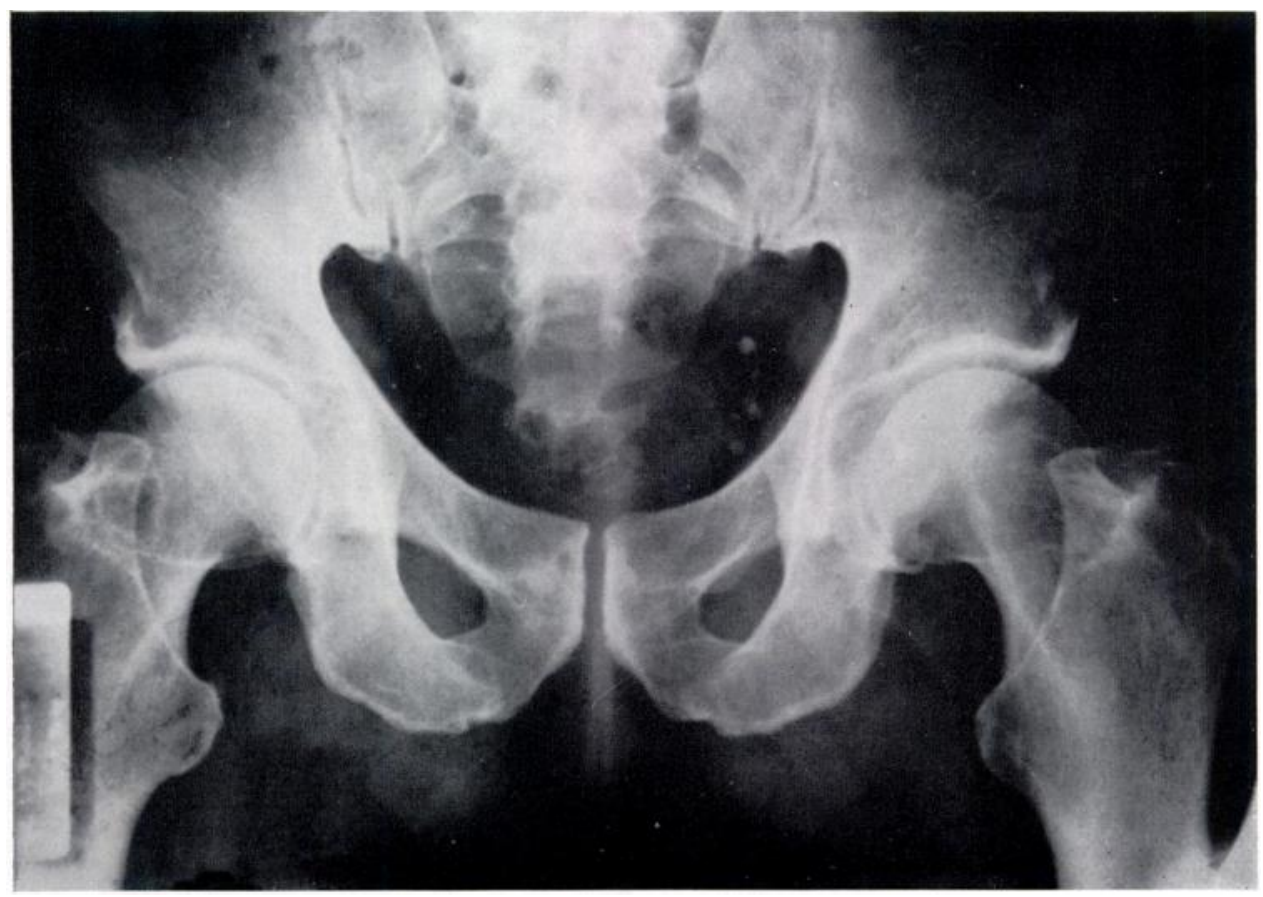

FIG. 1

Initial radiograph (undated) showing a clear symphysis pubis and normal hip joints.

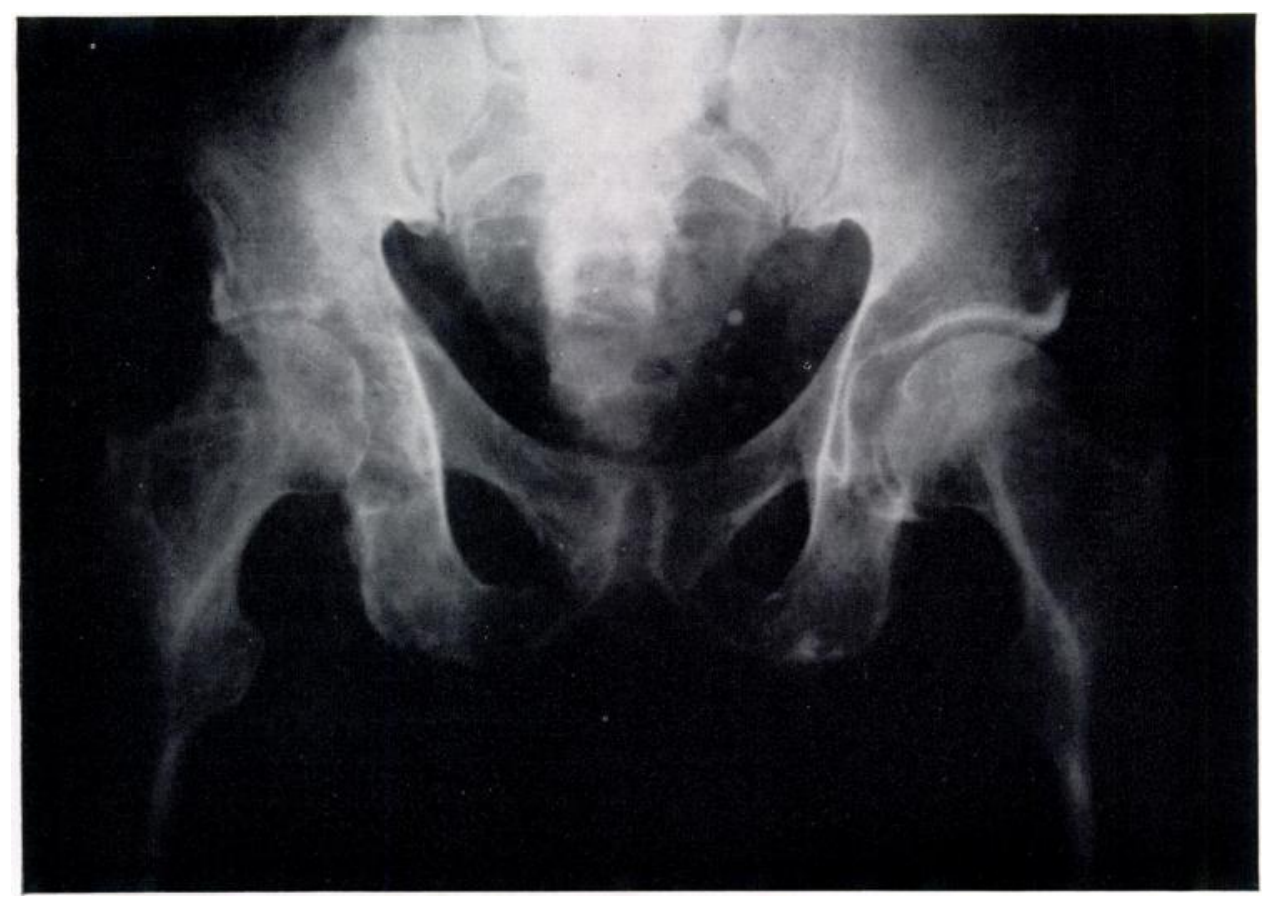

FIG. 2

Seven months after prostatectomy. Osteitis pubis with fusion of symphysis and early involvement of right hip joint. 


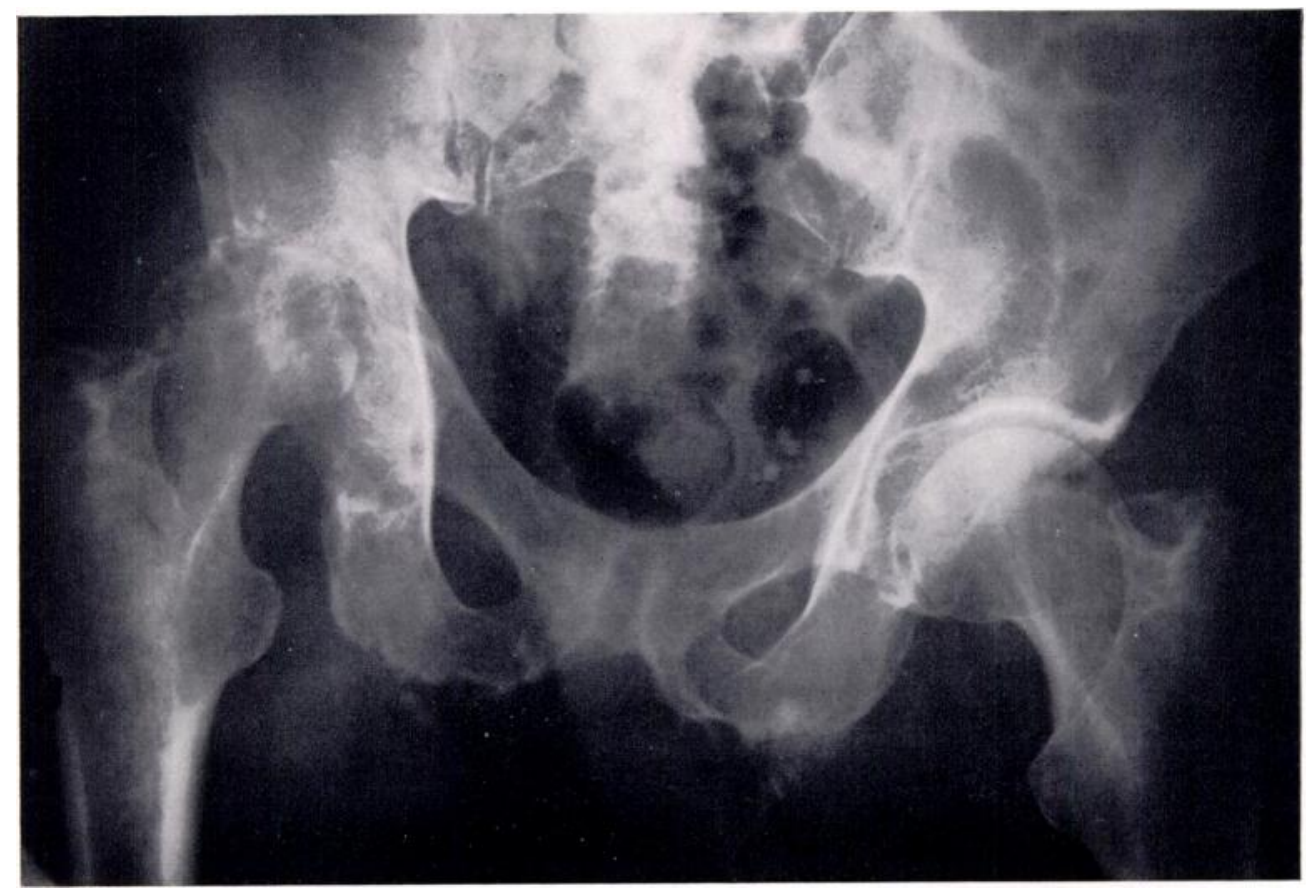

FIG. 3

Two months later. Severe involvement of right hip with loss of joint space and migration of acetabulum.

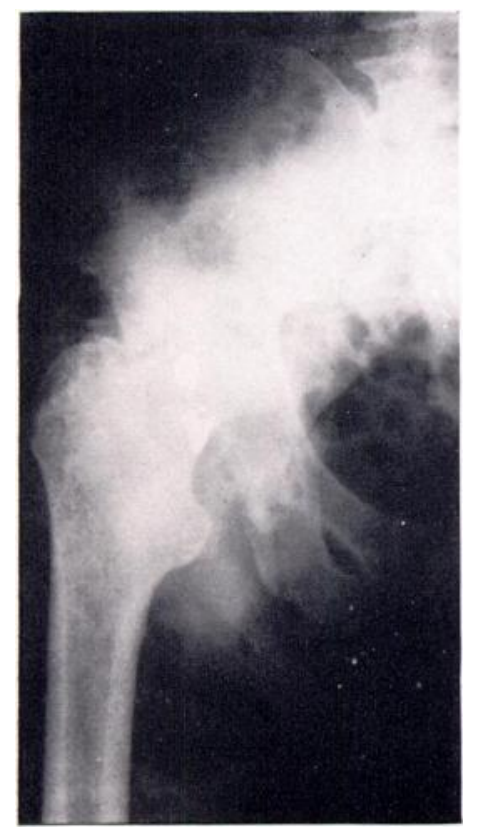

FIG. 4

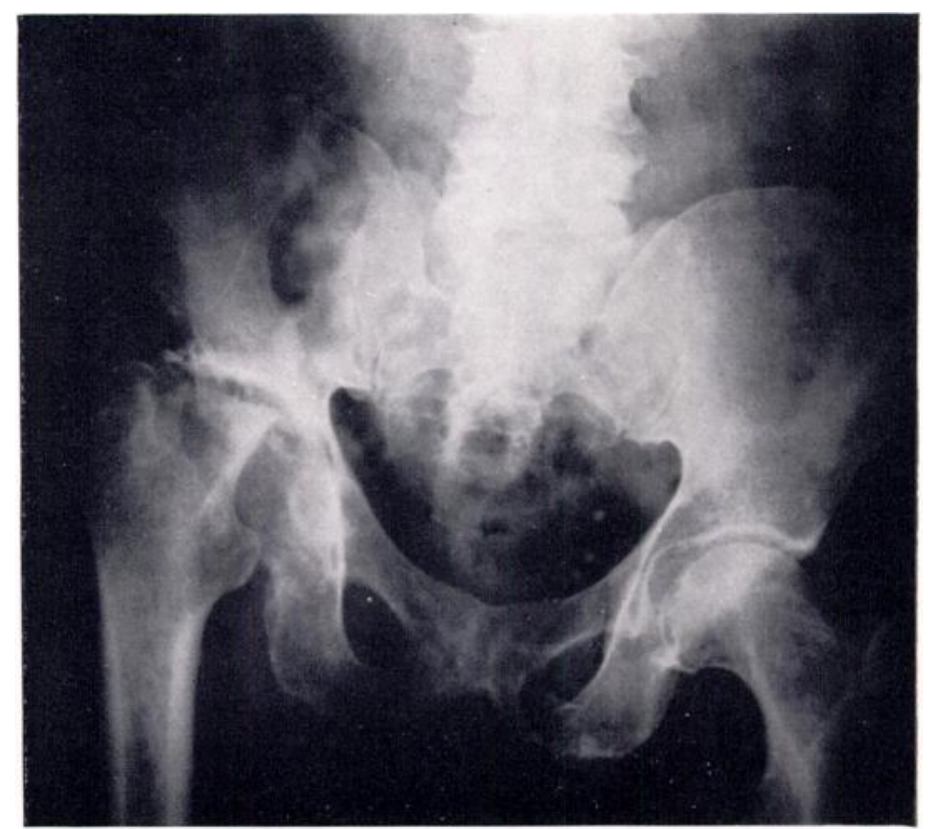

FIG. 5

Figure 4-Four months later still (one year after prostatectomy and six months after onset of disease in hip). There is moderate absorption of the femoral head. Figure 5-A year later (two years after prostatectomy). Stage of healing in right hip joint following absorption of femoral head. 
by Silver (1941). Each of these affections had followed suprapubic operations on the bladder or prostate. In Friedenberg's patient a biopsy showed infection with pseudomonas, for which antibiotics were given; but the destructive process progressed and an extra-articular arthrodesis was eventually performed. In Silver's two cases the disease was arrested without operation.

The cause of osteitis pubis is uncertain: injury with vaso-neurotrophic disorder, and infection with organisms of low virulence, have been suggested (Götzen and Boeminghaus 1953, Abrams, Sedlezky and Stearns 1949). The course of the disease in our patient strongly supports an infective origin in his case, even though no specific organism was isolated. Nevertheless, as in the cases described by Abrams et al. (1949), antibiotics did not seem to be helpful.

A self-limited course with healing in one situation and spread in another was observed in the present case both clinically and radiologically. Finally the activity of the hip lesion subsided, permitting moderate function despite severe destruction of the femoral head. It seems likely that in future cases of hip involvement satisfactory natural healing might be expected-as in uncomplicated osteitis pubis-if the joint be maintained in a good position while attention is paid to the general health of the patient.

\section{SUMMARY}

1. A case of arthritis of hip complicating osteitis pubis after retropubic prostatectomy is described.

2. It seems likely that bacterial infection was responsible in this case, although it was not proved.

3. Antibiotics did not appear to influence the course of the disease.

4. The progress of the disease was followed clinically and radiologically to its conclusion by natural healing with absorption of the femoral head, leaving a painless joint with good function.

We wish to thank Dr J. Cairney, Director-General of Health, New Zealand, for permission to publish this case report.

\section{REFERENCES}

Abrams, M., Sedlezky, I., and Stearns, D. B. (1949): Osteitis Pubis. New England Journal of Medicine, 240, 637.

FrIEDENBerG, Z. B. (1950): Osteitis Pubis with Involvement of the Hip Joint. Journal of Bone and Joint Surgery, 32-A, 924.

Götzen, F. J., and Boeminghaus, H. (1953): Über die Ostitis pubis. Zentralblatt für Chirurgie, 78, 1.

Silver, C. M. (1941): Pelvic Bone Changes Following Suprapubic Prostatectomy. Bulletin of the Hospital for Joint Diseases, 2, 10. 